\title{
Cultura organizacional y satisfacción laboral en los trabajadores de la Universidad Marcelino Champagnat
}

Mg. Wilder Javier Rojas Vásquez

Mg. Úrsula Yolanda Távara Zegarra

\section{RESUMEN}

La presente investigación tuvo como objetivo establecer la relación entre la cultura organizacional y la satisfacción laboral en los trabajadores de la Universidad Marcelino Champagnat. El diseño fue correlacional no causal. La población fue de 124 quienes eran la mayoría de los trabajadores de la Universidad Marcelino Champagnat los que contestaron el Inventario de Cultura Organizacional RST y la Escala de Satisfacción Laboral RST. Estos instrumentos fueron sometidos a juicio de expertos y pruebas de validación. Los resultados demostraron que se halló una relación entre cultura organizacional y satisfacción laboral $(r=.672 ; p=.000)$, así como entre sus dimensiones: carisma marista - normas institucionales y las dimensiones de satisfacción laboral, mostrando que existe una relación altamente significativa. Se concluye que existe una relación directa y significativa entre cultura organizacional y satisfacción laboral. Así mismo existe relación entre la dimensión de carisma marista que ha ayudado a mantener las normas institucionales y que permiten un buen ambiente de trabajo. Cada una de estas dimensiones contribuye a desarrollar la identidad institucional la que se refleja en la comunicación, la valoración del trabajo, los tipos de relaciones interpersonales, el bienestar laboral y las condiciones laborales del personal que labora en la universidad.

PALABRAS CLAVES: Cultura organizacional, satisfacción laboral, universidad privada, docentes y administrativos. 


\section{PLANTEAMIENTO DEL PROBLEMA}

Uno de los grandes desafíos que enfrentan las instituciones en la actualidad es mantener fidelidad a los principios que les dieron origen. La universidad como institución pasa por la misma experiencia pues los inicios nos muestran a casas de estudios identificadas con determinados valores que con el paso del tiempo van tomando otras formas de expresarse.

Es el interés de los investigadores relacionar la cultura organizacional con la satisfacción laboral y describir cómo el trabajador comparte sus creencias y valores con el personal que labora y con los estudiantes de la institución, lo que repercutirá en su actitud y desempeño.

Se cree que esto ayudaría a entender la relación entre el nivel de desempeño laboral y su actitud hacia la institución, su entorno y sus compañeros de trabajo.

En el caso de la Universidad Marcelino Champagnat aspectos positivos de la cultura interna como son la confianza, la cercanía, la sencillez, el trabajo autónomo, el espíritu de familia, siguen siendo una propuesta vivida a lo largo de estos años de servicio que tiene la institución. Lo que la universidad pretende es mantener la identidad con los valores propios, es decir, evitar que estos se diluyan en el tiempo o no formen parte de aquello que se considera esencial en la formación que la Universidad brinda.

La universidad Marcelino Champagnat tuvo durante sus primeros 20 años la presencia de quien fuera el hermano fundador, quien ya no estuvo presente en la etapa de crecimiento y expansión de la universidad. Esta influencia es muy importante al inicio de las organizaciones y va transformándose a medida que los diferentes miembros de la institución adquieren experiencia y encuentran sus propias soluciones.

El interés de esta investigación es demostrar la existencia de los valores en la cultura de la institución y la relación que ellos tienen con la satisfacción que los trabajadores experimentan por estar en la universidad. El trabajador, al vivir la experiencia de autoeficacia personal y que sus valores personales tienen coincidencia con su trabajo, se siente profesionalmente satisfecho.

Un aspecto muy importante son las relaciones personales, tanto en el centro de trabajo como en el entorno, pues en muchos casos son las que determinan la satisfacción e insatisfacción del individuo y su influencia en la cultura organizacional.

\subsection{Formulación del problema}

\subsubsection{Problema general}

La presente investigación plantea como problema: ¿Cuál es la relación entre la cultura organizacional y la satisfacción laboral en los trabajadores de la Universidad Marcelino Champagnat?

\subsection{Justificación}

A nivel teórico, la importancia de investigar la relación entre cultura organizacional y satisfacción laboral radicó en conocer y describir la relación entre la cultura organizacional y la satisfacción 
laboral del personal que trabaja en la Universidad Marcelino Champagnat debido a que no existen estudios previos.

A nivel práctico, este estudio fue importante porque de los resultados obtenidos se fortalece la responsabilidad de las personas comprometidas lo cual consolida la identidad de la institución y la encamina al logro de sus objetivos, hacia la calidad, pues cada organización es única, muy al margen de sus estructuras formales, ya que todo grupo humano desarrolla sus características particulares, lo que determinaría el aporte de la institución. Así mismo esta investigación fue transcendente, pues aportó a la identidad y al desarrollo axiológico de sus trabajadores lo que se podrá plasmar en talleres de inducción o jornadas que tengan por contenido los valores de la Universidad.

A nivel metodológico la presente investigación fue importante porque aportó a la construcción de un instrumento especialmente diseñado para medir la cultura organizacional y otro instrumento para medir la satisfacción laboral, lo cual es un aporte a la psicometría propia del país.

\section{MARCO TEÓRICO}

\subsection{Cultura organizacional y satisfacción laboral}

A continuación revisaremos los fundamentos teóricos de la cultura organizacional y la satisfacción laboral para lo cual se abordarán definiciones, características y teorías. En primer lugar, se desarrollan las conceptualizaciones teóricas de cultura organizacional y en segundo lugar de la satisfacción laboral.

\subsubsection{Definiciones sobre cultura organizacional}

Mencionaremos algunas definiciones sobre cultura organizacional:

Chiavenato (1989) presenta la cultura organizacional como un modo de vida, un sistema de creencias y valores, una forma aceptada de interacción y relaciones habituales de una determinada organización.

Robbins (1996) define que: "La cultura organizacional se refiere a un sistema de significados compartidos entre los miembros de una organización y que distingue a una de las otras" (p.681).

Así mismo Aguirre (2004) indica que la cultura organizacional es un conjunto de elementos interactivos fundamentales generados y compartidos por los miembros de una organización al tratar de conseguir la misión que da sentido a su existencia.

Después de revisar las definiciones que diversos especialistas han brindado consideramos que la definición más completa y sólida es la que propone Chiavenato (1989), por ello diremos que en la cultura organizacional las personas identificadas con la organización y las funciones que en ellas se cumplen inspirando al resto de los compañeros al compromiso que todo hombre debe tener, compartir funciones en equipo y sentimientos unificados.

En el caso de la universidad "Marcelino Champagnat" los valores se ven reflejados en el lema "SuperPetramEdificabo", lo que significa edificar sobre la roca. Quiere transmitir que los valores son la base para la formación de la persona, también posee un lema inspirado en las palabras 
del fundador de la congregación, quien dice que "para educar hay que amar"; es una muestra del deseo de acompañar la formación desde el interior de la persona. Estos son claros ejemplos de lo que construye la cultura de la institución.

\subsubsection{Características de cultura organizacional}

A continuación es importante que abordemos las características de la cultura organizacional. Al respecto Robbins (1987) propone siete características que deben ser consideradas dentro de una empresa, que al ser combinadas revelan la esencia de la cultura de la entidad:

- Autonomía Individual: Es el grado de responsabilidad, independencia y oportunidades para ejercer la iniciativa que las personas tiene en la organización.

- Estructura: Es el grado de normas y reglas, así como la cantidad de supervisión directa que se utiliza para vigilar y controlar el comportamiento de los empleados.

- Apoyo: Es el grado de ayuda y afabilidad que muestran los gerentes a sus subordinados.

- Identidad: Es el grado en que los miembros se identifican con la organización en su conjunto y no con su grupo o campo de trabajo.

- Desempeño-premio: Es el grado en que la distribución de premios dentro de la organización (aumento salarial, promociones), se basa en principios relativos al desempeño.

- Tolerancia al conflicto: Es el grado de conflicto presente en las relaciones de compañeros y grupos de trabajo, como el deseo de ser honestos y francos ante la diferencia.

- Tolerancia al riesgo: Es el grado en que se alienta al empleado para que sea innovador y corra riesgos.

Por su parte Chiavenato (2009) presenta seis características que definen a la cultura organizacional de las empresas en América.

a. Comportamiento observado con regularidad: cuando los miembros de una organización interactúan unos con otros, siempre usan un lenguaje y terminología común y realizan rituales relacionados con el comportamiento y la cortesía dentro de la organización.

b. Normas: en todas las organizaciones existen normas de comportamiento incluyendo directrices de cuánto trabajo tiene que hacer cada empleado.

c. Principales valores: los valores más importantes en una organización son aquellos que se defienden y se compartan entre todos los integrantes de una organización, estos pueden ser alta calidad en los productos, alta eficiencia, bajo ausentismo por parte de los empleados, etc.

d. Filosofía: políticas que establecen la creencia de cómo la organización tiene que tratar a los empleados 0 al cliente.

e. Reglas: estrictas directrices relacionadas con llevarse bien dentro de la organización. Los recién contratados tienen que aprender estas reglas con el fin de ser aceptados dentro de ella. 
f. Clima Organizacional: la percepción individual que tiene cada uno de los integrantes acerca de las características o cualidades de su organización. Esto es un sentimiento general que se desprende de la disposición física, la forma en que los participantes interactúan, y se comportan con los clientes o con otros agentes externos los miembros de la organización. Las empresas más admiradas tienen una fuerte cultura organizacional no hay que dejar de lado que el desempeño financiero juega un papel muy importante en los valores culturales de la organización y en como otros la perciben.

\subsubsection{Teorías de cultura organizacional}

A continuación señalaremos la teoría de la cultura organizacional que nos permitirán tener una mayor comprensión acerca de la variable de estudio:

En la Teoría de Chiavenato (2004) menciona que la cultura organizacional es parecida a un iceberg donde en la parte superior o sobre el nivel del agua se encuentran los aspectos visibles y superficiales observados en las organizaciones; se encuentran en esta parte la estructura física como edificios, los espacios, los muebles, la tecnología, métodos, políticas, etc. En la parte inferior se encuentran los aspectos invisibles y profundos, como aspectos psicológicos y sociológicos de la cultura.

Mientras mayor profundidad exista habrá más arraigo, por lo tanto la dificultad para cambiar la cultura será mucho mayor; el estrato superior, compuesto por aspectos físicos, es mucho más sencillo de modificar.

Se considera que el planteamiento de la teoría de Chiavenato, es la más acertada sobre la cultura organizacional, ya que sirve de marco de referencia a los miembros de la organización y da las pautas acerca de cómo las personas deben conducirse en esta.

Por otro lado, se analizará la teoría de cultura organizacional de Robbins(1993) quien afirma que se transmite a los trabajadores de diversas formas, en las que encontramos los relatos de casos, los rituales, los símbolos, los materiales y lenguaje. La cultura organizacional cumple varias funciones en el seno de una organización. En primer lugar, cumple la función de definir los límites; es decir, los comportamientos difieren unos de otros. Segundo, trasmite un sentido de identidad a sus miembros. Tercero, facilita la creación de un compromiso personal con algo más amplio que los intereses egoístas del individuo. Cuarto, incrementa la estabilidad del sistema social. La cultura es el vínculo social que ayuda a mantener unida a la organización al proporcionar normas adecuadas de los que deben hacer y decir los empleados.

El planteamiento de teoría de cultura organizacional de Robbins nos dice que la cultura organizacional permite la cohesión de las conductas individuales y la unificación con la visión empresarial para lograr los objetivos organizacionales y que las funciones específicas son desempeñar un papel que defina limites, es decir, que señale diferencias entre una organización y otra; confiere a la empresa un sentido de identidad; facilitar la generación del compromiso con algo superior al interés personal de cada empleado; reforzar la estabilidad del sistema social. 


\subsubsection{Definiciones de satisfacción laboral}

Después de haber revisado las conceptualizaciones teóricas sobre la cultura organizacional es necesario que abordemos los conceptos relacionados a la satisfacción laboral.

En primer lugar se analizará algunas definiciones que plantearon especialistas sobre la satisfacción laboral:

Para Loitegui (1990) la satisfacción laboral es un constructo pluridimensional que depende tanto de las características individuales del sujeto cuanto de las características y especificidades del trabajo que realiza. Además, el concepto de satisfacción en el trabajo está integrado por un conjunto de satisfacciones específicas, o aspectos parciales, que determinan la satisfacción general. Así entendida, la satisfacción laboral es una reacción afectiva general de una persona en relación con todos los aspectos del trabajo y del contexto laboral; es una función de todas las facetas parciales de la satisfacción. Este modelo de satisfacción implica un modelo compensatorio, de forma que un nivel elevado de satisfacción, en un determinado aspecto, puede compensar, o incluso suplir, otras deficiencias y carencias que en otras facetas laborales puedan producirse (p. 83).

Así mismo Strauss y Sayles (1992) definen la satisfacción laboral, como los sentimientos que el trabajador tiene hacia su trabajo, es una herramienta muy importante que los empleadores deben conocer, ya que obedece a múltiples factores tanto del trabajador como del trabajo en sí mismo. Por lo tanto la satisfacción en el trabajo es el resultado de diversas actitudes ante factores tales como: incentivos económicos, condiciones de trabajo, relaciones de mando, estilos gerenciales, relaciones interpersonales y características individuales.

Mientras que Robbins (1996) define la satisfacción laboral, centrándose básicamente, en los niveles de satisfacción e insatisfacción sobre la proyección actitudinal de positivismo 0 negativismo, como la actitud general de un individuo hacia su trabajo.

A su vez Márquez (2001) define que la satisfacción laboral, es una actitud del trabajador frente a su trabajo, dicha actitud está basada en las creencias y valores que el trabajador desarrolla de su propio trabajo. Las actitudes son determinadas conjuntamente por las características actuales del puesto como por las percepciones que tiene el trabajador de lo que "deberían ser".

De todas las definiciones expuestas se tomará el planteamiento de Robbinsque define la satisfacción laboral como el conjunto de actitudes generales del individuo hacia su trabajo. Ya que se acepta que quien está muy satisfecho con su puesto tiene actitudes positivas hacia éste; quien está insatisfecho, muestra en cambio, actitudes negativas. Esta definición tiene la ventaja de considerar la satisfacción como un proceso aprendido, que se desarrolla a partir de la interrelación dialéctica entre las particularidades subjetivas del trabajador y las características de la actividad y del ambiente laboral en general.

\subsubsection{Características de satisfacción laboral}

A continuación se analiza las principales características que presenta la satisfacción laboral, que han sido abordados por Robbins: 
Robbins (1996) indica que las características más importantes que conducen a la satisfacción laboral son: un trabajo desafiante desde el punto de vista mental, recompensas equitativas, condiciones de trabajo que constituyen un respaldo, colegas que apoyen y el ajuste personalidad - puesto. Por otra parte el efecto de la satisfacción en el puesto en el desempeño del empleado implica y comporta satisfacción y productividad, satisfacción y ausentismo y satisfacción y rotación.

En esta manifestación se encuentran características importantes que retroalimentan de forma positiva o negativa el desempeño de un puesto, conduciendo la satisfacción a una mejora en la productividad, a una permanencia estática o a una exigencia de movilidad, bien transitoria o definitiva, para satisfacer los niveles de exigencia personales.

\subsubsection{Teorías de satisfacción laboral}

La teoría que presentamos sobre la satisfacción laboral que consideramos en nuestro estudio.

Robbins (1998) en su teoría de satisfacción laboral considera que los principales factores que determinan la satisfacción laboral son:

- Reto del trabajo: los empleados tienen la tendencia a preferir trabajos que les oportunidad de usar sus habilidades y que favorezcan una variedad de tareas, libertad y retroalimentación de cómo se están desempeñando. Estas características hacen que un puesto sea mentalmente desafiante. Los trabajos que tienen muy poco desafío provocan aburrimiento pero un reto demasiado grande crea frustración y sensación de fracaso. En condiciones de reto moderado, la mayoría de los empleados experimentara placer y satisfacción.

- Sistema de recompensas justas: Los empleados requieren un sistema de salario y políticas de ascensos justos, sin ambigüedades y acordes a sus expectativas.

Cuando el salario se ve como justo con base en las demandas de trabajo, el nivel de habilidad del individuo y los estándares de salario de la comunidad, se favorece la satisfacción. La clave del enlace del salario con la satisfacción no es la cantidad absoluta que uno recibe, sino la percepción de justicia. De igual manera los individuos que perciben que las decisiones de ascenso se realizan con rectitud y justicia, probablemente experimentan satisfacción en sus trabajos.

- Condiciones favorables de trabajo: Los empleados se interesan en un ambiente de trabajo tanto para el bienestar personal como para facilitar el hacer un buen trabajo. Los estudios demuestran que los empleados prefieren ambientes físicos que no sean peligrosos o incomodos. La temperatura, la luz, el ruido y otros factores ambientales no deberían estar tampoco en el extremo, por ejemplo tener, demasiado calor o muy poca luz. Además la mayoría de empleados prefiere trabajar relativamente cerca de casa, en instalaciones limpias y más o menos modernas, con herramientas y equipo adecuado.

- Colegas que brinden apoyo: Para la mayoría de los empleados, el trabajo también cubre la necesidad de interacción social. Por tanto tener compañeros amigables que brinden apoyo lleve a una mayor satisfacción en el trabajo. Elcomportamiento del jefe es 
uno de los principales determinantes de la satisfacción. La satisfacción en el empleado se incrementa cuando el jefe es comprensivo, amigable y ofrece halagos por buen desempeño, escucha la opinión de los empleados y muestra un interés personal por ellos.

Acompañado de la compatibilidad entre personalidad y el puesto de trabajo.

Se acuerda tomar el planteamiento de la teoría de la satisfacción laboral de Robbins, ya que es importante para las organizaciones que un empleado tenga un alto nivel de satisfacción laboral demostrando satisfacción y lealtad hacia la institución; mientras que un empleado con insatisfacción tiene emociones desfavorables hacia su trabajo.

\section{OBJETIVO GENERAL}

\section{Generales}

Establecer la relación entre la cultura organizacional y la satisfacción laboral en los trabajadores de la Universidad Marcelino Champagnat.

\section{Específicos}

- Determinar el nivel cultura organizacional en los trabajadores de la Universidad Marcelino Champagnat.

- Determinar el nivel de satisfacción laboral en los trabajadores en la Universidad Marcelino Champagnat

- Establecer la relación entre carisma marista y normas institucionales con las dimensiones de satisfacción laboral en los trabajadores en la Universidad Marcelino Champagnat.

- Establecer la relación entre identidad Institucional y las dimensiones de satisfacción laboral en los trabajadores en la Universidad Marcelino Champagnat.

- Establecer la relación entre niveles de comunicación y las dimensiones de satisfacción laboral en los trabajadores en la Universidad Marcelino Champagnat.

\section{METODOLOGÍA}

\subsection{Diseño de investigación}

Es de tipo no experimental transversal, pues no se manipularán las variables de estudio. Así mismo, el diseño es correlacional no causal - bivariable, (Hernández, et al.,2014) dado que busca establecer la relación entre las variables de cultura organizacional y satisfacción laboral.

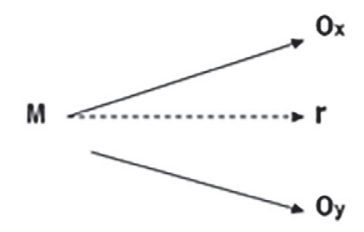

Donde :

$\mathrm{M}=$ muestra ( Población total)

0 = observación de la variable

$r$ relación 


\subsection{Variables a comparar}

Dado que se trata de una investigación no experimental, no existen variables independientes ni dependientes, sino que las variables de estudio son atributivas, porque no pueden ser manipuladas. (Kerlinger\& Lee, 2005, Sierra 2005, Alarcon 1991) en el sentido experimental del término.

A continuación se señalará la operacionalización de las variables de estudio:

Tabla 1. Operacionalización de las Variables cultura organizacional y satisfacción laboral

\begin{tabular}{|c|c|c|c|c|}
\hline Variable & Definición nominal & Dimensiones & Indicadores & Ítems \\
\hline $\begin{array}{c}\text { Cultura } \\
\text { organizacional }\end{array}$ & $\begin{array}{l}\text { Es "un modo de vida, un sistema de creencias } \\
\text { y valores, una forma aceptada de interacción } \\
\text { y relaciones típicas de determinada } \\
\text { organización."(Chiavenato, 1989). }\end{array}$ & $\begin{array}{l}\text { Carisma Marista y } \\
\text { Normas } \\
\text { Institucionales } \\
\text { Identidad } \\
\text { Institucional } \\
\text { Niveles de } \\
\text { Comunicación }\end{array}$ & $\begin{array}{l}.874 \\
.837 \\
.887\end{array}$ & $\begin{array}{l}8 \\
5 \\
8\end{array}$ \\
\hline $\begin{array}{l}\text { Satisfacción } \\
\text { Laboral }\end{array}$ & $\begin{array}{l}\text { Es como la actitud general de un individuo } \\
\text { hacia su trabajo. Robbins (1996). }\end{array}$ & $\begin{array}{l}\text { El ambiente de } \\
\text { trabajo } \\
\text { Tipo de } \\
\text { relaciones } \\
\text { interpersonales } \\
\text { Valoración } \\
\text { del trabajo } \\
\text { desarrollado } \\
\text { Bienestar laboral } \\
\text { Condiciones } \\
\text { laborales }\end{array}$ & $\begin{array}{l}.862 \\
.835 \\
.922 \\
.706 \\
.826\end{array}$ & $\begin{array}{l}5 \\
5 \\
9 \\
4 \\
6\end{array}$ \\
\hline
\end{tabular}

\subsection{Población y muestra}

La población estuvo conformada por 124 personas, todas ellas laboran en la Universidad Marcelino Champagnat. El tipo de muestreo es no probabilístico porque es el objetivo de estudio y porque la investigación ameritó tomar toda la población.

Tabla 2. Distribución de la población según género

\begin{tabular}{|l|c|c|}
\hline & Frecuencia & Porcentaje \\
\hline Masculino & 67 & 54.0 \\
\hline Femenino & 57 & 46.0 \\
\hline Total & 124 & 100.0 \\
\hline
\end{tabular}


Tabla 4. Distribución de la población según nivel académico

\begin{tabular}{|l|c|c|}
\hline & Frecuencia & Porcentaje \\
\hline Bachiller & 7 & 5.6 \\
\hline Licenciado & 31 & 25.0 \\
\hline Maestro & 39 & 31.5 \\
\hline Doctor & 13 & 10.5 \\
\hline Secundaria incompleta & 3 & 2.4 \\
\hline Secundaria completa & 9 & 7.3 \\
\hline Carrera técnica & 22 & 17.7 \\
\hline Total & 124 & 100.0 \\
\hline Dictibucion de la pobacon
\end{tabular}

Distribución de la población según tiempo de vinculación

\begin{tabular}{|l|c|c|}
\hline & Frecuencia & Porcentaje \\
\hline Menos de 5 años & 44 & 35.5 \\
\hline Entre 5 y 10 años & 34 & 27.4 \\
\hline Entre 10 y 15 años & 20 & 16.1 \\
\hline Entre 15 y 20 años & 13 & 10.5 \\
\hline Más de 20 años & 13 & 10.5 \\
\hline Total & 124 & 100.0 \\
\hline
\end{tabular}

\section{INSTRUMENTO}

\subsection{Inventario de cultura organizacional (RST)}

Se elaboró el Inventario de Cultura Organizacional tomando como base la teoría de Chiavenato y el Ideario de la Universidad Marcelino Champagnat. Se construyó el Inventario de Cultura Organizacional RST con el objetivo general de evaluar la cultura organizacional de la Universidad Marcelino Champagnat, teniendo como objetivos específicos identificar la espiritualidad y carisma marista, reconocer las normas y principios éticos, reconocer la identidad de los trabajadores con la universidad e identificar los niveles de comunicación, para lo cual se definen tres dimensiones las que se colocaron directamente en el instrumento, estas son:

\section{a. Carisma marista y Normas Institucionales}

Se entiende como Carisma Marista es el don dado por Dios a la Iglesia para la santificación y es tomado por un grupo específico de personas que se vinculan en una congregación o Instituto religioso.

Las normas institucionales: "la observancia general de dichas convenciones es una condición necesaria para el orden del mundo en que vivimos". Por eso creemos que mantener el orden en la institución es también un pilar importante en nuestra cultura organizacional. 


\section{b. Identidad institucional}

La identificación con la organización y sus objetivos es la variable clave que brinda las bases para un aumento del rendimiento tanto en las tareas pautadas como en un amplio abanico de conductas deseables.

\section{c. Niveles de comunicación}

La organización y la comunicación se encuentran estrechamente vinculadas para el funcionamiento de la empresa que busca resultados, dicho vínculo posee dos componentes: el político que son medios de una estrategia global en la que influyen y el sociocultural que son los valores, las representaciones y fenómenos de poder pesan para la organización y la comunicación.

\subsection{Escala de satisfacción laboral (RST)}

La construcción de la Escala de Satisfacción Laboral se tomó algunas dimensiones presentadas por Robbins (2001) en su libro Administración de Recursos Humanos. Tiene por objetivo general evaluar la satisfacción laboral de la universidad, consideramos los siguientes objetivos específicos; reconocer la valoración del ambiente de trabajo, identificar los tipos de relaciones interpersonales, reconocer la valoración del trabajo desarrollado, reconocer los niveles de desempeño profesional y reconocer las condiciones laborales, para lo cual hemos definidos cinco factores, que son:

\section{a. Ambiente de trabajo.}

Es el conjunto de condiciones que rodea a la persona y que directa o indirectamente influyen en su salud y su vida laboral.

\section{b. Tipo de relaciones interpersonales.}

Las relaciones interpersonales constituyen, un aspecto básico en las vidas de los trabajadores, funcionando no solo como un medio para alcanzar determinados objetivos sino como un fin en sí mismo.

\section{c. Valoración del trabajo.}

En el transcurso de la rutina laboral acontecen numerosos momentos profesionales, donde se viven momentos de aciertos profesionales y errores en las tareas. Si bien normalmente existe una normativa interna tendiente a contemplar o saber qué hacer frente a errores profesionales; es también de gran importancia a hacer y reconocer la actividad profesional cuando se producen a ciertos o muestras de valor agregado.

\section{d. Bienestar laboral}

El Bienestar laboral posee un carácter vivencial positivo, que surge del disfrute personal, consecuencia del grado óptimo de satisfacción del trabajador como expresión de la evaluación cognitiva -valorativa y resultado del balance entre las expectativas y los logros en el ámbito laboral, y que están considerablemente influenciados por la personalidad. Es la promoción y mantenimiento del más alto grado de bienestar físico, mental y social de los trabajadores en todas las ocupaciones. 


\section{e. Condiciones laborales.}

Son aquellas que existen objetivamente en los puestos y áreas de trabajo pero son percibidas por los trabajadores en función de sus necesidades individuales y de las características del trabajo que desarrollan. Es por esto que al estudiar la satisfacción de los trabajadores con las condiciones de trabajo debe valorarse la percepción que estos tienen sobre las mismas.

Para verificar la validez de contenido, se aplicó el método de criterio de jueces, que hicieron revisión detallada del contenido por ítems, con el objetivo de determinar si el lenguaje utilizado es apropiado y responde a cada dimensión de la Escala de Satisfacción Laboral RST.

Se seleccionó un grupo de 8 expertos en el tema materia de investigación, a quienes se les entregó una ficha de valoración solicitándoles la aprobación o desaprobación de los ítems. Para la determinación de la validez se utilizó el Coeficiente V. de Aiken, obteniéndose en la dimensión 1 El Ambiente de Trabajo se obtuvo que de los 4 ítems que conforman, 3 de ellos presentan una V. de 1.00 y 1 alcanzó una V. de .88. En la dimensión de Tipos de Relaciones Interpersonales presentan una V. de 1.00. En la dimensión de Valoración del Trabajo Desarrollado se obtuvo que de los 7 ítems que conforman 5 de ellos presentan una $V$. de 1.00 mientras los otros 2 ítems alcanzaron una V. de .88. En la dimensión Niveles de Desempeño Profesional todos los ítems presentan una V. de 1.00. De la misma manera, en la dimensión de Condiciones Laborales se obtuvo que de los 11 ítems que conforman 9 de ellos presentan una V. de 1.00 mientras que los 2 restantes alcanzaron una $\mathrm{V}$. de .88 , determinando de esta manera la validez de los ítems.

\section{RESULTADOS}

El gráfico 1, muestra los porcentajes de las categorías: bajo, medio, alto, evidenciando un 29.1 $\%$ que considera que existe una alta cultura organizacional en la Universidad Champagnat, un $45.2 \%$ que considera que la cultura organizacional es media. En tanto que el $25.8 \%$ considera baja cultura organizacional.

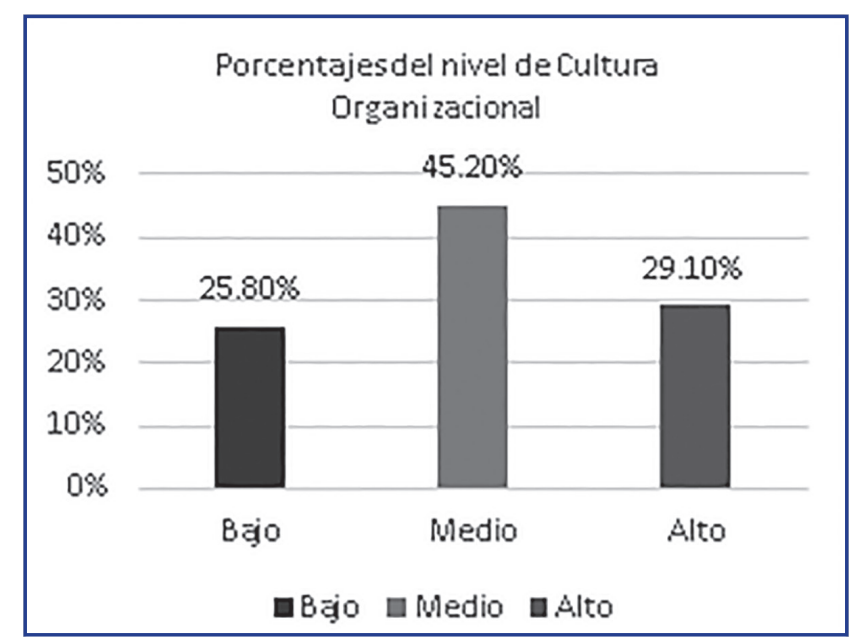

Gráfico 1 Porcentajes del nivel de Cultura Organizacional 
El gráfico 2, muestra los porcentajes acumulados en las categorías bajo, medio y alto. Presenta un $26.7 \%$ de satisfacción laboral alta, un $47.6 \%$ de satisfacción laboral media y un $25.8 \%$ de satisfacción laboral es bajo no se encuentra satisfecho.

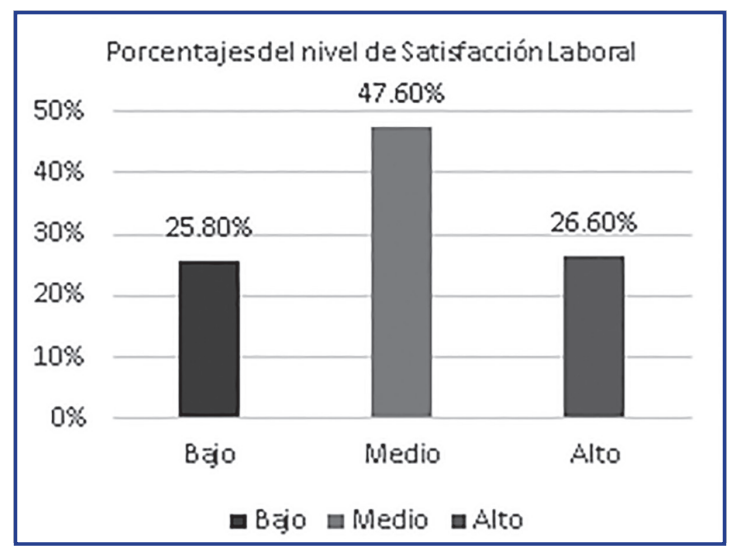

Gráfico 2 Porcentajes del nivel de Satisfacción Laboral

Para determinar si los datos presentan una distribución normal se utilizó la prueba de KolmogorovSmirnov. Como se observa en la tabla 18, ninguna de las dos variables presentan distribución normal, puesto que las probabilidades de significancia son menores de .05. Por lo tanto, la correlación se analizará a través del Coeficiente Rho de Spearman.

Tabla 1 Evaluación de la normalidad de las variables cultura organizacional y satisfacción laboral

\begin{tabular}{|l|c|c|}
\hline & Z de Kolmogorov-Smirnov & P \\
\hline CULTURA ORGANIZACIONAL & $.255^{\star \star *}$ & .000 \\
\hline SATISFACCIÓN LABORAL & $.271^{\star \star \star}$ & .000 \\
\hline Carisma Marista y Normas Institucionales & $.150^{\star \star \star}$ & .000 \\
\hline Identidad Institucional & $.220^{\star \star \star}$ & .000 \\
\hline Niveles de Comunicación & $.110^{\star \star \star}$ & .000 \\
\hline El Ambiente de trabajo & $.213^{\star \star \star}$ & .000 \\
\hline Tipo de Relaciones Interpersonales & $.216^{\star \star \star}$ & .000 \\
\hline Valoración del Trabajo desarrollado & $.165^{\star \star \star}$ & .000 \\
\hline Bienestar Laboral & $.146^{\star \star \star}$ & .000 \\
\hline Condiciones Laborales & $.130^{\star \star \star}$ & .000 \\
\hline
\end{tabular}

${ }^{* \star *}$ Altamente significativa $(p<.001)$

En la tabla 19 se muestra la relación entre carisma marista - normas institucionales y las dimensiones de satisfacción laboral. A partir de un coeficiente de correlación no paramétrico (Rho de Spearman) igual a .532 para ambiente de trabajo, $r=.620$ para tipo de relaciones interpersonales, $r=$ 
.613 para valoración del trabajo desarrollado, $r=.358$ para bienestar laboral y $r=.448$ para condiciones laborales, siendo para todos los casos el nivel de significancia $p<.001$, se puede afirmar que existe una relación positiva y directa entre carisma marista-normas institucionales y satisfacción laboral, a mayor carisma marista-normas institucionales existe mejor percepción del ambiente de trabajo, mejor tipo de relaciones interpersonales, existe una mejor valoración del trabajo desarrollado, bienestar laboral y condiciones laborales. Además esta relación es altamente significativa, por tanto se rechaza la hipótesis nula.

Tabla 2. Relación entre carisma marista - normas institucionales y las dimensiones de satisfacción laboral

\begin{tabular}{|l|l|c|c|c|c|c|}
\hline \multicolumn{2}{|c|}{} & $\begin{array}{c}\text { El Ambiente } \\
\text { de Trabajo }\end{array}$ & $\begin{array}{c}\text { Tipo de } \\
\text { Relaciones } \\
\text { Interpersonales }\end{array}$ & $\begin{array}{c}\text { Valoración } \\
\text { del Trabajo } \\
\text { desarrollado }\end{array}$ & $\begin{array}{c}\text { Bienestar } \\
\text { Laboral }\end{array}$ & $\begin{array}{c}\text { Condiciones } \\
\text { Laborales }\end{array}$ \\
\hline $\begin{array}{l}\text { Carisma Marista } \\
\text { y Normas } \\
\text { Institucionales }\end{array}$ & Rho & $.532^{\star \star}$ & $.620^{\star \star}$ & $.613^{\star \star}$ & $.358^{\star \star}$ & $.448^{\star \star}$ \\
\cline { 2 - 7 } & $\mathrm{P}$ & .000 & .000 & .000 & .000 & .000 \\
\hline
\end{tabular}

${ }^{* *}$ Correlación significativa $(p<.001)$

En la tabla 20, se muestra la correlación entre la dimensión identidad institucional y las dimensiones de Satisfacción laboral. Con un coeficiente de correlación no paramétrica (Rho de Spearman) de .507 para ambiente de trabajo, $r=.466$ para tipo de relaciones interpersonales, $r=.514$ para valoración del trabajo desarrollado, $r=.295$ para bienestar laboral y $r=.346$ para condiciones laborales, siendo en todos los casos el nivel de significancia $p<.001$, se puede afirmar que existe una relación positiva directa es decir, a mayor Identidad Institucionales existe mayor Satisfacción laboral. Por tanto, se rechaza la hipótesis nula.

Tabla 3. Relación entre identidad institucional y las dimensiones de satisfacción laboral

\begin{tabular}{|l|l|c|c|c|c|c|}
\hline \multicolumn{2}{|c|}{} & $\begin{array}{c}\text { El Ambiente } \\
\text { de Trabajo }\end{array}$ & $\begin{array}{c}\text { Tipo de } \\
\text { Relaciones } \\
\text { Interpersonales }\end{array}$ & $\begin{array}{c}\text { Valoración } \\
\text { del Trabajo } \\
\text { desarrollado }\end{array}$ & $\begin{array}{c}\text { Bienestar } \\
\text { Laboral }\end{array}$ & $\begin{array}{c}\text { Condiciones } \\
\text { Laborales }\end{array}$ \\
\hline $\begin{array}{l}\text { Identidad } \\
\text { Institucional }\end{array}$ & Rho & $.507^{\star *}$ & $.466^{\star *}$ & $.514^{\star *}$ & $.295^{\star *}$ & $.346^{\star *}$ \\
\cline { 2 - 7 } Sig. (bilateral) & $\begin{array}{l}\text { Sig. } \\
\text { (bilateral) }\end{array}$ & .000 & .000 & .000 & .001 & .000 \\
\hline
\end{tabular}

${ }^{* *}$ correlación significativa $(p<.001)$

En la tabla 21, se muestra la correlación entre la dimensión niveles de comunicación y las dimensiones de satisfacción laboral. Con un coeficiente de correlación no paramétrica de $r=.537$ para ambiente de trabajo, $r=.711$ para tipo de relaciones interpersonales, $r=660$ para valoración del trabajo desarrollado, $r=.360$ para bienestar laboral y $r=.505$ para condiciones laborales, 
todos con un nivel de significancia de $p=.000$. Por lo tanto, se puede afirmar que existe una relación positiva directa es decir, a mayor nivel de comunicación existe mayor satisfacción laboral. Por tanto, se rechaza la hipótesis nula.

Tabla 4. Relación entre niveles de comunicación y las dimensiones de satisfacción Laboral

\begin{tabular}{|l|l|l|l|l|l|l|}
\hline \multicolumn{2}{|l|}{} & $\begin{array}{c}\text { El Ambiente } \\
\text { de Trabajo }\end{array}$ & $\begin{array}{c}\text { Tipo de } \\
\text { Relaciones } \\
\text { Interpersonales }\end{array}$ & $\begin{array}{c}\text { Valoración } \\
\text { del Trabajo } \\
\text { desarrollado }\end{array}$ & $\begin{array}{c}\text { Bienestar } \\
\text { Laboral }\end{array}$ & $\begin{array}{l}\text { Condiciones } \\
\text { Laborales }\end{array}$ \\
\hline \multirow{2}{*}{$\begin{array}{l}\text { Niveles de } \\
\text { Comunicación }\end{array}$} & Rho & $.537^{\star \star}$ & $.711^{\star \star}$ & $.660^{\star \star}$ & $.360^{\star \star}$ & $.505^{\star \star}$ \\
\cline { 2 - 7 } & $\begin{array}{l}\text { Sig. } \\
\text { (bilateral) }\end{array}$ & .000 & .000 & .000 & .000 & .000 \\
\hline
\end{tabular}

${ }^{* *}$ correlación significativa $(p<.001)$

La tabla 22 muestra la correlación de cultura organizacional y satisfacción laboral, con un coeficiente de correlación no paramétrica de $r=.672$ y un nivel de significancia de $p=.000$ para ambos. Se puede afirmar que existe una correlación positiva directa es decir, existe una relación entre cultura organizacional y satisfacción laboral. Por tanto, se rechaza la hipótesis nula.

Tabla 5. Relación entre cultura organizacional y satisfacción laboral

\begin{tabular}{|llc|}
\hline \multicolumn{2}{|c|}{ SATISFACCIÓN LABORAL } \\
\hline Rho & $P$ \\
\hline CULTURA ORGANIZACIONAL & $.672^{\star * *}$ & .000 \\
\hline
\end{tabular}

${ }^{* * *}$ correlación significativa $(p<.001)$

\section{DISCUSIÓN RESULTADOS}

La discusión de nuestra investigación la hemos organizado bajo el esquema que nos presenta Hernández et al. (2014) quien nos sugiere plantear la evaluación de las implicancias que ha tenido la investigación, luego se ha relacionado los resultados obtenidos con estudios existentes para reconocer así las limitaciones de estudio. Una de las limitaciones para la investigación fue el recolectar la información al momento de aplicar las pruebas a los trabajadores de la Universidad Marcelino Champagnat pues no todos contaban con el tiempo que requería para completar los cuestionarios. Previamente era necesario planificar el día y aun así se debía esperar que el personal se desocupara para poder atender al grupo.

En el análisis de la relación de las variables de estudio cultura organizacional y satisfacción laboral, se determinó que existe una correlación directa y significativa $(r=.672, p=.000)$ para ambos. Al respecto, Chiavenato (1989) nos dice, que la Cultura Organizacional es un modo de vida, un sistema 
de creencias y valores, una forma aceptada de interacción y relaciones típicas de determinada organización, esto quiere decir que la Cultura Organizacional es la médula de la organización que está presente en todas las funciones y acciones que realizan todos sus miembros.

Asimismo, Robbins (1996) afirma que la satisfacción laboral es una actitud general de un individuo hacia su trabajo. Podemos describirla como una disposición psicológica del sujeto hacia su trabajo (lo que piensa de él), y esto supone un grupo de actitudes y sentimientos. De ahí que la satisfacción o insatisfacción con el trabajo dependa de numerosos factores como el ambiente físico donde trabaja, el hecho de que el jefe lo llame por su nombre y lo trate bien, el sentido de logro 0 realización que le procura el trabajo, la posibilidad de aplicar sus conocimientos, que el empleo le permita desarrollar nuevos conocimientos y asumir retos, etc.

Murillo, Calderón y Torres (2003) concluyeron que el nivel de satisfacción laboral de los trabajadores y la repercusión en la cultura demuestran que la satisfacción del trabajador está asociada con culturas orientadas al empleado, corporativistas, abiertas, pragmáticas. Esto explicaría porque la cultura organizacional está ligada a una adecuada satisfacción laboral. En nuestro caso de estudio hemos observado esta relación directa y significativa pues los trabajadores reconocen la importancia de la axiología presente en su cultura organizacional lo que ayuda a tener una percepción también significativa en relación a la satisfacción laboral.

En el análisis de la variable cultura organizacional existe un $29.1 \%$ del personal de la universidad que percibe una alta cultura organizacional, un $45.2 \%$ que considera que la cultura organizacional es de nivel medio y el $25.8 \%$ considera nivel bajo la cultura organizacional. Al respecto estos resultados se relacionan con lo que dice Fernández (2002) a quien presentamos en nuestros antecedentes sobre cultura organizacional, quien llega a la conclusión que el concepto de cultura organizacional es válido y fructífero para un estudio sociológico de organizaciones educativas. En nuestro caso se aplicaría al nivel que obtienen los trabajadores de la Universidad pues la mayoría está en un porcentaje de $45.2 \%$ lo que muestra que hay una cierta cultura, pero que aún debe ser trabajada, pues en el caso específico de la universidad no existe un programa sistematizado sobre cultura organizacional.

Con respecto al análisis de la variable satisfacción laboral muestra un $26.7 \%$ de satisfacción laboral en nivel alto, un $47.6 \%$ de satisfacción laboral en nivel medio, y un $25.8 \%$ de satisfacción laboral en nivel bajo, es decir, no se encuentra satisfecho. Si comparamos con el estudio de Navarro (2008) quien afirma que los factores de satisfacción laboral más importantes son los relacionados con la naturaleza y el contenido del trabajo.

Así mismo, Merino y Díaz (2008) realizaron un estudio sobre el nivel de satisfacción laboral en las empresas públicas y privadas. Obteniendo como resultado tres niveles de satisfacción, alto, medio y bajo para las dimensiones que investigaron, las cuales fueron la satisfacción con su trabajo, con compañeros y jefes, con la remuneración y las capacitaciones. En general el nivel de satisfacción laboral en las empresas públicas y privadas de Lambayeque se encuentra en un nivel medio y bajo, dado que tan solo el 30\% presentó un nivel alto de satisfacción. Este resultado coincide con la presente investigación en la que podemos observar que al igual que estos autores en su 
investigación, la relación con jefes y compañeros influye en los niveles de satisfacción, así como las remuneraciones y las capacitaciones.

Esto explicaría los resultados obtenidos en la encuesta y se concluye que las personas se sienten satisfechas en el trabajo cuando disfrutan del mismo y describen globalmente su experiencia laboral en términos positivos.

Para el análisis de la relación entre carisma marista - normas institucionales y las dimensiones de satisfacción laboral se determinó que existe una correlación no paramétrica $(r=532)$ para ambiente de trabajo, $(r=.620)$ para tipo de relaciones interpersonales, $(r=.630)$ para valoración del trabajo desarrollado, $(r=.613)$ para bienestar laboral y $(r=.644)$ para condiciones laborales, siendo para todos los casos el nivel de significancia $p<.001$. Se puede afirmar que existe una relación positiva y directa entre carisma marista-normas institucionales y las dimensiones de satisfacción laboral, a mayor carisma marista-normas institucionales existe mejor percepción del ambiente de trabajo, mejor tipo de relaciones interpersonales, existe una mejor valoración del trabajo desarrollado, bienestar laboral y condiciones laborales. Además esta relación es altamente significativa.

Por ello concluimos que el marco axiológico de una institución influye en las normas institucionales como se presenta en el Plan estratégico elaborado por la Universidad Marcelino Champagnat el año 2012. Para Sammon (2006) el carisma de los hermanos maristas es inspirado por su fundador debido a la presencia viva del Espíritu Santo. Asimismo, Chiavenato (2009) manifiesta que las normas institucionales influyen en las directrices de cuánto trabajo tiene que hacer cada empleado y son condiciones necesarias para mantener el orden en la institución en un ambiente de trabajo y contribuyen a una positiva percepción de la institución.

Esto explicaría por qué existe esta relación entre el carisma marista - normas institucionales y las dimensiones de satisfacción laboral; con lo cual nuestra hipótesis de significancia queda comprobada; ya que los trabajadores valoran el Carisma Marista dentro de su institución.

De acuerdo con Robbins (1987) la valoración por el trabajo depende del grado en que los miembros se identifican con la organización en su conjunto. Esto lo confirma Chiavenato (1989) que sustenta que las personas identificadas con la organización comparten funciones en equipo y sentimientos unificados.

Así mismo Ollarves (2006) en su estudio sobre la Cultura Organizacional y propiedades motivantes del puesto de trabajo en una institución de Educación Superior en Venezuela, determino el grado de motivación de los docentes de acuerdo a la labor que desempeñan.

De esta manera, se puede afirmar que carisma marista -normas institucionales está ligada a las dimensiones de satisfacción Laboral, esto muestra que en la medida que el trabajador se identifica con la organización y sus objetivos nos permite reconocer la actividad profesional y su relación con las condiciones laborales valorando la percepción que tienen sobre las mismas.

Así mismo, para la dimensión identidad institucional y las dimensiones de satisfacción laboral. Se halló una correlación no paramétrica $(r=.507)$ para ambiente de trabajo, $(r=.466)$ para tipo 
de relaciones interpersonales, $(r=.514)$ para valoración del trabajo desarrollado, $(r=.295)$ para bienestar laboral y $(r=.346)$ para condiciones laborales, siendo en todos los casos el nivel de significancia $p<.001$, se puede afirmar que existe una relación positiva directa es decir, a mayor identidad institucionales existe mayor satisfacción laboral. Se relaciona con lo que sostiene Contreras (2006) en cuya investigación demostró que los elementos necesarios: comunicación interna, clima organizacional, valores y creencias organizacionales son necesarios para detallar la personalidad de la empresa y describirla como una organización con una cultura fuerte.

En el caso de la Universidad Marcelino Champagnat, la identidad y las dimensiones de satisfacción laboral muestran una relación significativa.

Según el análisis de los resultados, en la dimensión niveles de comunicación y las dimensiones de satisfacción laboral se halló una correlación no paramétrica de $(r=.537)$ para ambiente de trabajo, $(r=.711)$ para tipo de relaciones interpersonales, $(r=660)$ para valoración del trabajo desarrollado, $(r=.360)$ para bienestar laboral y $(r=.505)$ para condiciones laborales, todos con un nivel de significancia de $(p=.000$.) Por lo tanto, se puede afirmar que existe una relación positiva directa es decir, a mayor Niveles de comunicación existe mayor Satisfacción laboral. Según Robbins (1998) manifiesta que el trabajo también cubre la necesidad de interacción social. El comportamiento del jefe es uno de los principales determinantes de la satisfacción. Esto debe estar acompañado con la personalidad y el puesto de trabajo.

Para Díaz (2001) cada parte de la organización refleja lo que está sucediendo en el todo organizacional. Así, la calidad de comunicaciones internas y externas sus deficiencias y limitaciones son el reflejo de lo que pasaba en su cultura organizacional.

En el caso de la universidad esta relación directa y significativa debe ser mantenida y además potenciar pues la calidad de la comunicación influye en las relaciones interpersonales, la que se puede ver afectada cuando no tiene una adecuada atención y puede influenciar en la percepción de bienestar laboral.

\section{CONCLUSIONES}

1. Existen una relación significativa entre Cultura Organizacional y Satisfacción Laboral siendo una correlación positiva directa. Con esto reconocemos que en la Universidad Marcelino Champagnat como institución con valores específicos está logrando la identificación de sus trabajadores.

2. Existe un nivel medio de cultura organizacional y satisfacción laboral en los trabajadores de la Universidad Marcelino Champagnat que se explica por el porcentaje mayoritario obtenido por medio del instrumento de recolección de datos.

3. Existen una relación positiva directa entre el carisma marista, las normas institucionales y las dimensiones de satisfacción laboral, esto se debe a la presencia constante del carisma en las diversas actividades, las que han influido en las normas y en el ambiente de trabajo que se percibe en la universidad.

4. Existe una relación significativa entre la dimensión identidad institucional con las dimensiones de satisfacción laboral en los trabajadores de la Universidad Marcelino Champagnat. 
5. Existe una relación significativa entre nivel de comunicación y las dimensiones de satisfacción laboral que se da por brindar canales permanente de comunicación, de cercanía entre personal de la Universidad Marcelino Champagnat.

\section{RECOMENDACIONES}

- Se recomienda difundir las políticas de desarrollo que promueve la Universidad Marcelino Champagnat y que le permita al personal experimentar un progreso profesional e involucrarse con la organización.

- Fomentar un programa sistematizado de cultura organizacional sostenido en el tiempo que permita profundizar en los valores propios de la universidad y que incentive en sus trabajadores la pertenencia e identidad.

- Es importante que la universidad trabaje en las dimensiones de satisfacción laboral pues son los aspectos que tienen menor valoración.

- Implementar beneficios sociales para que se conviertan en una estrategia organizacional que permita satisfacer las expectativas del personal y lograr una conducta positiva a la organización.

- Diseñar programas de ascenso y un escalafón que proporcionen retos y competencia entre trabajadores para que se motiven en realizar sus actividades y ser sobresalientes dentro de la institución. De esta manera los trabajadores se sentirán satisfechos y realizados profesionalmente.

- Se recomienda hacer una revisión de las formas de las relaciones interpersonales y de la comunicación en los trabajadores si bien son positivas hay aspectos de comunicación que se deben mejorar.

- Se recomienda programas de formación para el personal de la Universidad que le permita sentirse identificado con la visión y misión que la Universidad promueve.

- Recomendamos mantener actividades de integración que fomenten el clima de fraternidad, espíritu de familia que es característica de una institución Marista ya que son los aspectos de mayor valoración en la investigación realizada.

- Recomendamos una especial atención en los programas de formación dirigido a aquellos grupos de menor nivel de formación pues son los que han obtenido menores puntajes dentro de la media de Cultura Organizacional y Satisfacción Laboral. 


\section{REFERENCIAS}

Abarbanel, H. (1997). Cultura organizacional: aspectos teóricos, prácticos y metodológicos. Bogotá, Colombia: Legis.

Aguirre, A. (2004). La Cultura de las organizaciones. Barcelona- España: Editorial Ariel.

Añez, S. (2006). Cultura organizacional y motivación laboral de los docentes universitarios. Universidad Dr. Rafael Belloso Chacín: Venezuela.

Álvarez, D. (2007). Satisfacción y fuentes de presión laboral y satisfacción laboral en docentes de universidades estatales y universidades privadas de Lima Metropolitana. Recuperado de http://www.redalyc.org/pdf/1471/147112813004.pdf.

Alarcón, R. (2008). Métodos y diseños de investigación del comportamiento. Lima: Fondo Editorial de la Universidad Ricardo Palma.

Baca, M., Lau, I. y Ríos, B. (2007). Evaluación y diagnóstico de la cultura organizacional de la Municipalidad Distrital de San Isidro. Universia. Recuperado de http://biblioteca.universia. net/html_bura/ficha/params/title/evaluacion-diagnostico-cultura-organizacional.

Barraza, M. A. y Ortega. M. F. (2009). Satisfacción laboral en instituciones formadoras de docentes. Un primer acercamiento. Ponencia presentada en el Congreso de Investigación Educativa, Veracruz, México.

Bartola, A. (1992). Comunicación y Organización. La organización comunicante y la comunicación organizada. Barcelona, España: Paidós.

Bayona, C., Goñi, S. y Madorrán, C. (2000). Estudio sobre compromiso organizacional: Implicaciones para la gestión estratégica de los recursos humanos. Revista Europea de Dirección y Economía de la Empresa,9 (1), 139-149.

Blum, M y James, N. (1982): Psicología Industrial ( Sus Fundamentos Teóricas y Sociales). Editorial Trillas. México.

Cavalcante, S. (2004). Satisfacciones en el trabajo de los directores de escuelas secundarias públicas de la Región de Jacobina,Bahía- Brasil. (Tesis doctoral). Universidad Autónoma de Barcelona.

Chiavenato, I. (1989). Teoría general de la administración (4ed.). Bogotá. Colombia: Mc Graw Hill.

Chiavenato, I. (2003). Administración de recursos humanos (6ed.). Bogotá, Colombia: Mc Graw Hill.

Chiavenato Idalberto (2009). Comportamiento organizacional. La dinámica del éxito en las organizaciones. ( $2^{\circ} \mathrm{ed}$.). Monterrey, México: Mc Graw Hill. 
Comisión Internacional Marista de Educación (1998). Misión Educativa Marista. Recuperado de http://www.champagnat.org/e_maristas/ Documentos/missaoEducativaMarista_ES.pdf

Contreras, 0. (2006). Cultura organizacional en el área administrativa de una institución privada de educación superior en Zacatecas. Razón y Palabra, 12 Recuperado de http://www.redalyc. org/pdf/1995/199520735027.pdf

Denison, D. (1991). Cultura Corporativa. Santa fé de Bogotá, Colombia: Legis

Díaz, G. Y. (2001). Desarrollo de la imagen institucional del Colegio Santa María de Matellini de Chorrillos a través de la planificación estratégica. (Tesis de Licenciatura). Universidad Mayor de San Marcos. Lima. Perú.

Edel, N., García, S y Casiano, B (2007). Clima y compromiso organizacionales. Vol. I versión electrónica gratuita. Recuperado de http.//eumed.net/libros/2007c/

Fernández, J. (2002).Cultura organizacional y centro educativo.(Tesis Doctoral). Universidad Complutense de Madrid, España.

Fernández, B y Paravic, T. (2003). Nivel de satisfacción laboral en enfermeras de hospitales públicos y privados de la provincia de Concepción en Chile. Ciencia y Enfermería IX, 9 (2) ,57-66.

Fuentes, L. J (2006). Incidencia de la satisfacción laboral docente y el ambiente de aula en el rendimiento académico de los estudiantes universitarios en Chile. (Tesis Doctoral). Universidad de Sevilla, España.

Gawel, Joseph E.(1997). Herzberg's theory of motivation and maslow's hierarchy of needs. Practical Assessment. Research \& Evaluation,5(11). Recuperado de http://PAREonline.net/ getvn.asp?v $=5 \& n=11$.

Gibson,J.et al.(1985). Organizaciones, conducta,estructura y proceso. Editorial Interamericana. México.

Guedez,V. (1998). Gerencia Cultura y Educación. Caracas: Fondo Editorial Tropykos/Clacdec.

Herzberg, F., Mausner, B., y Snyderman, B. B. (1959). The motivation to work (2nd ed.). New York: John Wiley\&Sons.

Hernández, S.T.(2010) Diagnóstico de satisfacción laboral en una empresa textil peruana. Hemeroteca. Recuperado de http://www.monografias.com/trabajos13/emtex/emtex.shtml.

Hernández, R., Fernández, C. y Baptista, P. (2014). Metodología de la investigación. México D.F.: McGraw-Hill Education.

Hueso, A. y Cascant, M.(2012). Metodología y técnicas cuantitativas de investigación. Universitat Politécnica de Valencia. Recuperado de https://riunet.upv.es/bitstream/handle/10251/17004/ 
Mg. Wilder Javier Rojas Vásquez / Mg. Úrsula Yolanda Távara Zegarra

Instituto de Hermanos Maristas (2007). Agua sobre la Roca. Roma.

Jiménez, M. (2009). Plan de mejora que favorezca el clima organizacional fundamentado en el análisis y gestión de la satisfacción laboral de los trabajadores en la Tienda el Fundador. Universidad Cien fuegos Carlos Rafael Rodríguez. Cuba. Kerlinger, F.\& Lee, H (2002). Investigación del comportamiento. México D.F: Mc Graw-Hill/ Interamericana.

Locke, E.A. (1976). The nature and causes of job satisfaction.In Dunnette. Handbook of industrial and organization a psychology. Chicago. United States: Rand McNally College.

Loitegui, J.R. ( 1990). Determinantes de las satisfacción laboral en empleados de la administración foral de Navarra. Tesis doctoral. Universidad Complutense de Madrid. España.

Marcelo, C. (2009) Valoración del trabajo realizado. Lima. Recuperado de http://www. creacionempresa.es/valoracion-de-un-trabajo-realizado/

Márquez, M. (2001). Satisfacción laboral. Recuperado de http://www.monografias.com/trabajos10/ sala/sala.shtml.

Marroquín, M y Villa, A. (1995). La comunicación interpersonal. Medición y estrategias para su desarrollo. Recursos e instrumentos psicopedagógicos.Bilbao, España: Mensajero.

Martínez, H.N y Dávila, A (1998). Pregunta y respuesta sobre un espacio vacío de investigación: El estudio de las culturas en las instituciones educativas. Revista Mexicana de Investigación Educativa, 3(6). Recuperado de http://www.redalyc.org/pdf/140/14000602.pdf

Meliá, J. L y Peiró, J. M (1998). Satisfacción Laboral S10/12. Recuperado de http://www. uv.seguridadlaboral.

Merino, M. y Díaz, A (2008). Nivel de satisfacción laboral en empresas públicas y privadas. Estudio empresariales. Recuperado: dialnet.unirioja.es/descarga/articulo/2710495.pdf

Morán, C. y Manga, D. (2005). Satisfacción en el trabajo, autoestima y stress: diferencias por ocupación y género. Recuperado de www.ruct.uva.es/pdf/Revista\%206/6115.pdf

Moreno, Ríos, Canto, San Martin y Perle (2010) Satisfacción laboral y burnout en trabajos poco cualificados. Diferencias entre sexos en población inmigrantes. Recuperado de http://www. redalyc. org/pdf/2313/231316502008.pdf

Murillo, S, Calderón, G y Torres, K (2003). La cultura organizacional y bienestar social.Cuadernos de Administración, 16 Recuperado dewww.redalyc.org/pdf/205/20502506.pdf

Navarro, E. (2008). Aportación al estudio de la satisfacción laboral de los profesionales técnicos del sector de la construcción: una aplicación cualitativa en la Comunidad Valenciana.(Tesis Doctoral). UniversidadPolitécnica de Valencia. España. 
Ollarves, Y (2006). Cultura Organizacional y propiedades motivantes del puesto de trabajo en una Institución de Educación Superior, 21 Recuperado de http://www.scielo.org.ve/scielo. php?script $=$ sci_arttext\&pid $=$ S1316-00872006000100006

Real Academia de la Lengua Española (2012). Diccionario. Madrid, España Recuperado http:// buscom.rae.es/drael/html/cabecera.

Robbins, S. (1996). Comportamiento organizacional. Concepto, controversias y aplicaciones. México, D-F: Prentice-Hall Hispanoamericana.

Robbins, S.\& De Cenzo (2001). Administración de Recursos humanos. México, D-F: LimusaWiley.

Robbins, S. (2004). Comportamiento Organizacional (10ma.ed.) México, D.F: Pearson.

Salgado, C. (2012). Efectos del Bienestar Espiritual sobre la Resiliencia en estudiantes universitarios de Argentina, Bolivia, Perú y República Dominicana (Tesis Doctoral). Universidad Nacional Mayor de San Marcos, Lima, Perú.

Sammon,.S (2006). Dar a conocer a Jesucristo y hacerlo amar- La vida apostólica maristahoy. Circulares, Vol. XXXI, n 3

Serna, H. (1997). Gerencia Estratégica: Planeación y Gestión - Teoría y Metodología. 3R. Editores. Bogotá.

Sierra Bravo, R (2005). Tesis doctorales y trabajos de investigación científica. Madrid: Thomson.

Silva, H. (2009). Medición del clima laboral y satisfacción del personal de trabajadores docentes y no docentes de la Facultad de Medicina de la Universidad Nacional de la Amazonía Peruana. Recuperado: http://www.unapiquitos.edu.pe/oficinas/iiunap/archivos/2009/ medicina/Art.Cientifico\%20Dr.\%20Hermann\%20Silva.doc

Schein, E. (1988). La cultura empresarial y el liderazgo. Barcelona, España: Plaza y Janes

Schein, E (1992). Sicología de la Organización. México, D.F: Prentice Hall.

Stoner,J y Freeman, E. (1994). Administración (5ta.ed.), México, D.F: Prentice Hall

Strauss, G y Sayles, L. (1992). Personal. México: Prentice Hall.

Topa, C.G y Morales, F. (2006). Identificación organizacional y productividad personal en grupos de trabajo: Un modelo de ecuaciones estructurales.Anales de Psicología, 22(2),234-242. Recuperado de http://www.redalyc.org/pdf/167/16722208.pdf

Universidad Marcelino Champagnat (2012). Plan estratégico. Lima: autor.

Valencia, P. (2008). Estilos gerenciales y satisfacción laboral (Tesis para optar el Grado de Magister en Administración). Universidad Nacional Mayor de San Marco, Lima, Perú. 
Mg. Wilder Javier Rojas Vásquez / Mg. Úrsula Yolanda Távara Zegarra

Vairrud R. (2003). Convertir sueños en realidad. Ediciones(IESA).Venezuela.

Velásquez, R. (2001). La satisfacción con las condiciones de trabajo. Una propuesta para su medición. Gestiopolis Recuperado de http://www.gestiopolis.com/canales/derrhh/ articulos/25/seghso.htm. 